\title{
Pathway-focused proteomic signatures in HER2-overexpressing breast cancer with a basal-like phenotype: New insights into de novo resistance to trastuzumab (Herceptin)
}

\author{
CRISTINA OLIVERAS-FERRAROS ${ }^{1,2^{*}}$, ALEJANDRO VAZQUEZ-MARTIN ${ }^{1,2^{*}}$, BEGOÑA MARTIN-CASTILLÓ $^{1,2^{*}}$, \\ MARIA CARMEN PÉREZ-MARTÍNEZ ${ }^{2,3}$, SILVIA CUFíl ${ }^{1,2}$, SONIA DEL BARCO ${ }^{1,2}$, LUIS BERNADO ${ }^{2,3}$, \\ JOAN BRUNET $^{1,2}$, EUGENI LÓPEZ-BONET ${ }^{2,3}$ and JAVÍER A. MENENDEZ ${ }^{1,2}$ \\ ${ }^{1}$ Catalan Institute of Oncology (ICO); ${ }^{2}$ Girona Biomedical Research Institute (IdIBGi); ${ }^{3}$ Department of Pathology, \\ Dr Josep Trueta University Hospital of Girona, Avenida de Francia S/N, E-17007 Girona, Catalonia, Spain
}

Received May 3, 2010; Accepted June 2, 2010

DOI: 10.3892/ijo_00000716

\begin{abstract}
Pioneering clinical studies in de novo refractoriness to the anti-HER2 monoclonal antibody trastuzumab have suggested that HER2 gene-amplification can take place also in a basal-like molecular background to generate basal/HER $2^{+}$tumors intrinsically resistant to trastuzumab. Here, we first investigated the unique histogenesis of the basal/HER $2^{+}$phenotype in breast carcinomas. The presence of basal CK5/CK6 cytokeratin expression in HER2 ${ }^{+}$tumors revealed a significant overlap in the histological features of HER $2^{+} / \mathrm{CK} 5 / 6^{+}$and basal-like breast carcinomas. Basal/ HER $2^{+}$tumors were typically poorly differentiated, highgrade invasive ductal carcinomas with large geographic necrosis, pushing margins of invasion, syncytial arrangement of tumor cells, ribbon- or festoon-like architecture, squamous metaplasia, stromal lymphocytic infiltrates, high mitotic index and strong p53 positivity. Secondly, we performed low-scale proteomic approaches in JIMT-1 cells, a unique model of HER2-gene amplified trastuzumab-resistant breast carcinoma with a basal-like phenotype, to develop biomarker signatures that may differentiate trastuzumab-responsive from non-responsive tumors. When applying antibody-based array technology to the extracellular milieu of trastuzumabrefractory JIMT-1 and trastuzumab-sensitive SKBR3 cell cultures, JIMT-1 cells were found to secrete higher amounts of several growth factors including amphiregulin, EGF, IGFBP-6, PDGF-AA, neurotrophins, TGFß and VEGF. Semiquantitative signaling node multi-target sandwich ELISAs
\end{abstract}

Correspondence to: Dr Javíer A. Menendez, Catalan Institute of Oncology, Girona (ICO-Girona), Dr Josep Trueta University Hospital, Ctra. França s/n, E-17007 Girona, Catalonia, Spain E-mail: jmenendez@iconcologia.net

${ }^{*}$ Contributed equally

Key words: HER2, basal-like, trastuzumab, insulin-like growth factor, proteomics revealed that JIMT-1 cells drastically overactivate RelA, the prosurvival subunit of $\mathrm{NF}-\kappa \mathrm{B}$ as compared to trastuzumabsensitive luminal/HER2 ${ }^{+}$SKBR3 cells. When simultaneously assessing the activation status of 42 receptor tyrosine kinases (RTK) using a human phospho-RTK array, JIMT-1 cells were found to constitutively display hyperactivation of the insulin-like growth factor-I receptor (IGF-1R). High-content immunofluorescence imaging revealed that activated IGF-1R mainly localized at focal adhesion-like structures in JIMT-1 cells. In vitro wound healing assays suggested that this functional reorganization of the JIMT-1 cytoskeletal reorganization may account for an exacerbated trastuzumab-refractory 'migratogenic' phenotype. Forthcoming studies should validate the notion that identification of basal-like immunophenotypes and/or basal-like molecular signatures within HER2 ${ }^{+}$breast carcinomas may provide rapid means to define subgroups of breast cancer patients likely to display resistance to trastuzumab ab initio.

\section{Introduction}

Clinical benefit of using the anti-HER2 monoclonal antibody trastuzumab (Herceptin) is limited by the fact that secondary (i.e. acquired) resistance develops rapidly (within 1 year) in most of trastuzumab-treated HER 2 gene-amplified breast cancer patients (1-4). On the other hand, $70 \%$ of HER2overexpressing metastatic breast carcinomas show primary resistance to trastuzumab as a single agent and approximately $15 \%$ of women diagnosed with early HER $2^{+}$disease are de novo resistant to trastuzumab and relapse in spite of treatment with trastuzumab-based therapies $(5,6)$. Although significant amount of pre-clinical and clinical research has been dedicated to elucidate molecular mechanisms that could explain the appearance of acquired resistance to trastuzumab (7-10), there have been few studies addressing the ultimate molecular mechanisms that could explain de novo (i.e. intrinsic or primary) resistance to trastuzumab (11-13). Indeed, HER2 status yet remains as the only available biomarker for selecting breast cancer patients for trastuzumab-based therapy. 
Pioneering findings by Harris and colleagues demonstrated that HER2-overexpressing tumors with a basal-like phenotype and/or with expression of insulin-like growth factor-I receptor (IGF-IR) and other proteins involved in growth factor pathways were more likely to be intrinsically resistant to pre-operative trastuzumab (14). Lesniak and colleagues recently demonstrated that overexpression of $B 1-$ integrin, an adhesion molecule involved in cell migration, invasion, spreading and signaling in prototypical basal-like breast carcinomas, is an independent negative prognostic factor for tumor progression of HER2 ${ }^{+}$metastatic breast cancer patients treated with trastuzumab-based chemotherapy (15). Although microarray studies have repeatedly classified HER2+ breast carcinomas as a molecular entity separate from basal-like tumors (16-18), the above-mentioned clinical findings strongly suggest that HER2 gene-amplification could take place also in a basal-like molecular background $(19,20)$.

Here, we sought to confirm and expand the clinical notion that a mixed basal/HER2 ${ }^{+}$molecular scenario may account for refractoriness to HER targeting therapies ab initio. First, we sought to confirm that histologic features of some HER2+ breast carcinomas significantly overlap those attributed solely to basal-like tumors $(21,22)$. Second, low-scale proteomic approaches were performed for biomarker discovery in cultured JIMT-1 cells, a unique model of HER2-gene amplified breast carcinoma with a basal-like phenotype (23).

\section{Materials and methods}

Immunohistochemistry. Breast cancer tissues including core biopsies and surgical specimens were analyzed for HER2, CK5/6, p53 using standardized immunohistochemical techniques and robotic autostainers (Dako Cytomation, Inc.). Briefly, 3- $\mu \mathrm{m}$ sections of the cancer tissue were placed on positively charged glass microscope slides. Deparaffinization involved incubation of the slides in xylene followed by graded alcohol series in a routine manner. Endogenous peroxidase activity was blocked with $3 \%$ hydrogen peroxidase and methanol. Samples were steamed for antigen retrieval with $10 \mathrm{mM}$ citrate buffer ( $\mathrm{pH} 6.0$ ) for $30 \mathrm{~min}$. Following protein block, slides were incubated with antibodies for ER, PR, HER2, p63 and CK5/6. Binding results were visualized with the Envision ${ }^{\circledR}$ System (Dako Cytomation, Inc.), which uses horseradish peroxidase-labelled polymer that is conjugated with secondary antibodies. 3,3'-diaminobenzidine tetrahydrochloride (DAB) was used for the visualization of the antibody/enzyme complex. Slides were counterstained with hematoxylin and examined by light microscopy. Tumor immunoreactivity was scored 0 , negative; 1 , weak positive; and 2 , moderate/strong positive in combination with the percent of cells showing positive staining.

Histopathology. Hematoxylin- and eosin-stained slides were evaluated under light microscopy. Breast carcinomas were evaluated for grade [1-3] using the modified Scarff-BloomRichardson grading system comprising an architectural grade, nuclear grade and mitotic grade. Mitotic counts were performed using the $\mathrm{x} 40$ objective on an Olympus $\mathrm{BH}-2$ microscope. Breast cancer tissues were also evaluated for tumor histologic type, presence of geographic necrosis, quantity of apoptotic cells, border appearance, lymphocytic stromal response, nucleoli, nuclear chromatin pattern, apocrine features, metaplastic features, large central acellular zone and medullary features.

Cell lines and culture conditions. SKBR3 human breast cancer cells were obtained from the American Type Culture Collection (ATCC) and they where routinely grown in improved MEM (IMEM; BioSource International; Invitrogen S.A., Barcelona, Spain) supplemented with $5 \%$ fetal bovine serum (FBS) and $2 \mathrm{mM}$ L-glutamine. JIMT-1 human breast cancer cell line was established at Tampere University and is available from the German Collection of Microorganisms and Cell Cultures (http://www.dsmz.de/). JIMT-1 cells were grown in F-12/DMEM (1:1) supplemented with 10\% FBS and $2 \mathrm{mM} \mathrm{L-glutamine.} \mathrm{Cells} \mathrm{were} \mathrm{maintained} \mathrm{at} 37^{\circ} \mathrm{C}$ in a humidified atmosphere of $95 \%$ air and $5 \% \mathrm{CO}_{2}$. Cells were screened periodically for Mycoplasma contamination.

Antibody-based arraying of growth factors/secreted growth factor receptors. To prepare conditioned media, SKBR3 and JIMT-1 cells were plated in 100-mm tissue culture dishes and cultured in regular media with 5\% FBS until they reached $75-80 \%$ confluence. Cells were washed twice with serumfree media, and incubated overnight in serum-free IMEM (SKBR3) or F-12/DMEM (JIMT-1). Cells were then cultured for $48 \mathrm{~h}$ in low-serum (i.e., $0.1 \% \mathrm{FBS}$ ) media. The supernatant were collected, centrifuged at 1,000 x g, aliquoted, and immediately stored at $-80^{\circ} \mathrm{C}$ until utilization. RayBio ${ }^{\circledR}$ human growth factors/secreted growth factor receptors array 1 was purchased from RayBiotech, Inc. (Norcross, GA, USA) and antibody-based arraying was carried out as per manufacturer's instructions. Briefly, membranes were blocked with 5\% BSA/TBS (0.01 M Tris $\mathrm{HCl}, \mathrm{pH} 7.6 / 0.15 \mathrm{M} \mathrm{NaCl})$ for $1 \mathrm{~h}$. Membranes were then incubated with $\sim 2 \mathrm{ml}$ of conditioned media prepared from SKBR3 and JIMT-1 cell lines after normalization with equal amounts of protein for $2 \mathrm{~h}$. After extensive washing with TBS/0.1\% v/v Tween-20 (3 times, 5 min each) and TBS ( 2 times, 5 min each) to remove unbound materials, the membranes were incubated with a cocktail of biotin-labeled antibodies against different individual cytokines/ metalloproteases. The membranes were then washed and incubated with HRP-conjugated streptavidin $(2.5 \mathrm{pg} / \mathrm{ml})$ for $1 \mathrm{~h}$ at room temperature. Unbound HRP-streptavidin was washed out with TBS/0.1\% Tween-20 and TBS. Finally the signals were detected by ECL system. Densitometric values of spots were quantified using Kodak Image Analysis Software (Kodak).

Semi-quantitative determination of AKT, Stat3, p38 MAPK, MEK1 and $N F-\kappa B$ phosphorylation status. CST's PathScan ${ }^{\circledR}$ Signaling Nodes Multi-Target Sandwich ELISA Kit no. 7272 was purchased from Cell Signaling Technology, Inc. This solid phase sandwich enzyme-linked ImmunoSorbent assay combines the reagents necessary to detect endogenous levels of AKT1, phospho-AKT1 (Ser473), phospho-MEK1 (Ser217/ 221), phospho-p38 MAPK (Thr180/Tyr182), phospho-Stat3 (Tyr705) and phospho-NF-кB p65 (Ser536). SKBR3 and JIMT-1 cells (75-80\% confluent) were starved overnight and 
then cultured in the absence or presence of $100 \mu \mathrm{g} / \mathrm{ml}$ trastuzumab in low-serum ( $0.1 \%$ FBS)-containing culture medium for $48 \mathrm{~h}$. Cells were washed twice with cold-PBS and then lysed in buffer [20 mM Tris $\mathrm{pH} 7.5,150 \mathrm{mM} \mathrm{NaCl}, 1 \mathrm{mM}$ EDTA, 1 mM EGTA, 1\% Triton ${ }^{\circledR} \mathrm{X}-100,2.5 \mathrm{mM}$ sodium pyrophosphate, $1 \mathrm{mM}$ ß-glycerolphosphate, $1 \mathrm{mM} \mathrm{Na}_{3} \mathrm{VO}_{4}$, $1 \mu \mathrm{g} / \mathrm{ml}$ leupeptin, $1 \mathrm{mM}$ phenylmethylsulfonylfluoride, and complete protease inhibitor cocktail (Sigma-Chemicals)] for $30 \mathrm{~min}$ on ice. The lysates were cleared by centrifugation in an Eppendorff tube $\left(15 \mathrm{~min}\right.$ at $\left.14,000 \mathrm{x} \mathrm{g}, 4^{\circ} \mathrm{C}\right)$. Protein content was determined against a standardized control using the Pierce Protein Assay Kit (Rockford, IL, USA). Differential phosphorylation of AKT1, phospho-AKT, phosphoMEK1, phospho-p38 MAPK, phospho-Stat3 and phospho$\mathrm{NF}-\kappa \mathrm{B}$ p65 was measured as per manufacturer's instructions. Briefly, after incubation with cell lysates at a protein concentration of $0.5 \mathrm{mg} / \mathrm{ml}$, the target phospho-protein is captured by the antibody coated onto the microwells. Following extensive washing, a detection antibody is added to detect the captured target phospho-protein. An HRP-linked secondary antibody is then used to recognize the bound detection antibody. The HRP substrate TMB is added to develop color. The magnitude of absorbance (measured at $450 \mathrm{~nm}$ ) for this developed color is proportional to the quantity of bound target protein. Statistical analysis for differential expression levels was carried out with XLSTAT (Addinsoft ${ }^{\mathrm{TM}}$ ) and $\mathrm{P}<0.01$ was considered to be significant.

Antibody array-based profiling of phospho-receptor tyrosine kinases (RTKS). SKBR3 and JIMT-1 cells were plated in $100-\mathrm{mm}$ tissue culture dishes and cultured in regular media containing $10 \%$ FBS until they reached $75-80 \%$ confluence. The cells were then washed twice with serum-free medium and incubated overnight upon serum-free conditions. Cells were then stimulated to grow in 5\% FBS-containing medium in the presence or absence of $100 \mu \mathrm{g} / \mathrm{ml}$ trastuzumab for $48 \mathrm{~h}$. Cells were rinsed with cold phosphate-buffered saline (PBS) and immediately solubilized in NP-40 lysis buffer [1\% NP-40, 20 mmol/l Tris-HCl (pH 8.0), 137 mmol/l NaCl, 10\% glycerol, $2 \mathrm{mmol} / \mathrm{l} \mathrm{EDTA}, 1 \mathrm{mmol} / \mathrm{l}$ sodium orthovanadate, $10 \mu \mathrm{g} / \mathrm{ml}$ aprotinin, $10 \mu \mathrm{g} / \mathrm{ml}$ leupeptin, $1 \mathrm{mmol} / 1$ phenylmethyl-sulfonylfluoride, and complete protease inhibitor cocktail (Sigma-Chemicals)] by rocking the lysates gently at $4^{\circ} \mathrm{C}$ for $30 \mathrm{~min}$. Following microcentrifugation at $14,000 \mathrm{x} \mathrm{g}$ for $5 \mathrm{~min}$, supernatants were transferred into a clean test tube and sample protein concentrations were determined using the Pierce Protein Assay Kit (Rockford, IL, USA). Lysate (500 $\mu \mathrm{g}$ ) was diluted and incubated with the Human Phospho-RTK Array Kit (Proteome Profiler; R\&D Systems, Minneapolis, $\mathrm{MN}$, USA) as per the manufacturer's instructions. Arrays data were developed on X-ray film following exposure to chemiluminescent reagents.

Immunofluorescence staining and high-content confocal imaging. Cells were seeded at approximately 5,000 cells/well in 96-well clear bottom imaging tissue culture plates (BectonDickinson Biosciences; San Jose, CA, USA) optimized for automated imaging applications. Triton X-100 permeabilization and blocking, primary antibody staining, secondary antibody staining using Alexa Fluor ${ }^{\circledR}$ 488/594 goat anti- rabbit/mouse IgGs (Invitrogen, Molecular Probes, Eugene, OR, USA) and counterstaining (using Hoechst 33258; Invitrogen) were performed following BD Biosciences protocols. Images were captured in different channels for Alexa Fluor 488 (pseudo-colored green), Alexa Fluor 594 (pseudocolored red) and Hoechst 33258 (pseudo-colored blue) on a BD Pathway ${ }^{\mathrm{TM}} 855$ Bioimager System (Becton-Dickinson Biosciences) with 20 or $x 40$ objectives (NA 075 Olympus). Both acquisition and merging of images were carried out according to the Recommended Assay Procedure using BDAttovision $^{\mathrm{TM}}$ software. We employed the following antibodies: 1:50 dilution of anti-PP-IGF-IR (Tyr 1161) (sc-101703; Santa Cruz Biotechnology, Santa Cruz, CA, USA), 1:40 dilution of Alexa Fluor 594 Phalloidin (A12381; Invitrogen), and 1:400 dilution of anti-vinculin (V9264, clone hVIN-1, Sigma-Aldrich, St. Louis, MO, USA)

Wound-healing motility assays. Cells were seeded onto sixwell dishes at $1 \times 10^{5}$ per well. A single scratch wound was created using a p10 micropipette tip into confluent cells. Cells were washed thrice with PBS to remove cell debris, supplemented with assay medium in the absence or presence of $100 \mu \mathrm{g} / \mathrm{ml}$ trastuzumab, and monitored. Images were captured by phase-contrast microscopy at 0,24 and $48 \mathrm{~h}$ after wounding.

\section{Results}

A subgroup of HER2-overexpressing breast carcinomas overlaps in the histologic features of the basal-like phenotype. Preliminary studies using formalin-fixed, paraffinembedded block tissues from the cancer tissue collection in our Department of Pathology at the Dr Josep Trueta University Hospital of Girona confirmed that ER/PR-negative HER2+ breast carcinomas exist that simultaneously express basal epithelium cytokeratins markers (e.g. CK5/CK6) $(21,24)$. In some cases, HER2 ${ }^{+}$breast tumors that stained positively for basal cytokeratins displayed a focal checkerboard pattern with CK5/CK6-negative and CK5/CK6-positive tumor cells located next to each other (Fig. 1A). Interestingly, some HER2+-positive tumors stained uniformly or almost uniformly with CK5/CK6, thus resembling pure basal-like breast carcinomas. Indeed, this subgroup of HER2 ${ }^{+}$breast carcinomas displayed histological features of basal-like tumors: They were typically poorly differentiated, high-grade invasive ductal carcinomas with large geographic necrosis, pushing margins of invasion, syncytial arrangement of tumor cells, ribbon- or festoon-like architecture, squamous metaplasia, stromal lymphocytic infiltrates, high mitotic index [ranging from 19 mitoses/10 high-power filed to 32 mitoses/10 highpower fields (average 28 mitoses/10 high-power fields)] and strong p53 positivity (Fig. 1B) $(22,25)$. Some of the histopathological features found in the basal/HER ${ }^{+}$subgroup closely resembled those previously reported in medullary (lymphocytic infiltrate and pushing margins) or metaplastic (squamous metaplasia) breast carcinomas, two rare types of breast cancer with a well-recognized basal-like immunohistochemical profile (26).

JIMT-1 breast cancer cells: A model for studying HER2overexpressing breast cancer with a basal-like phenotype. In 


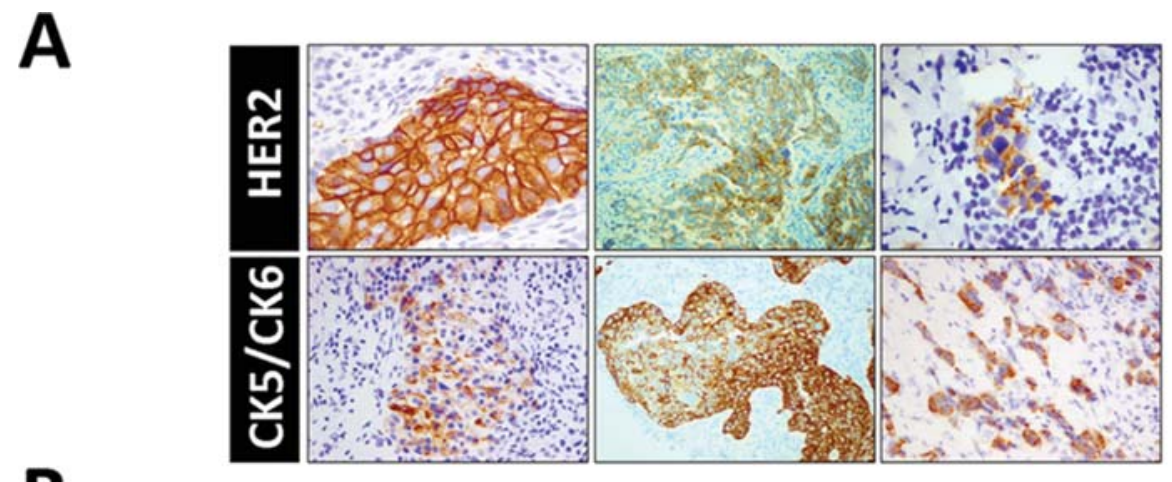

B
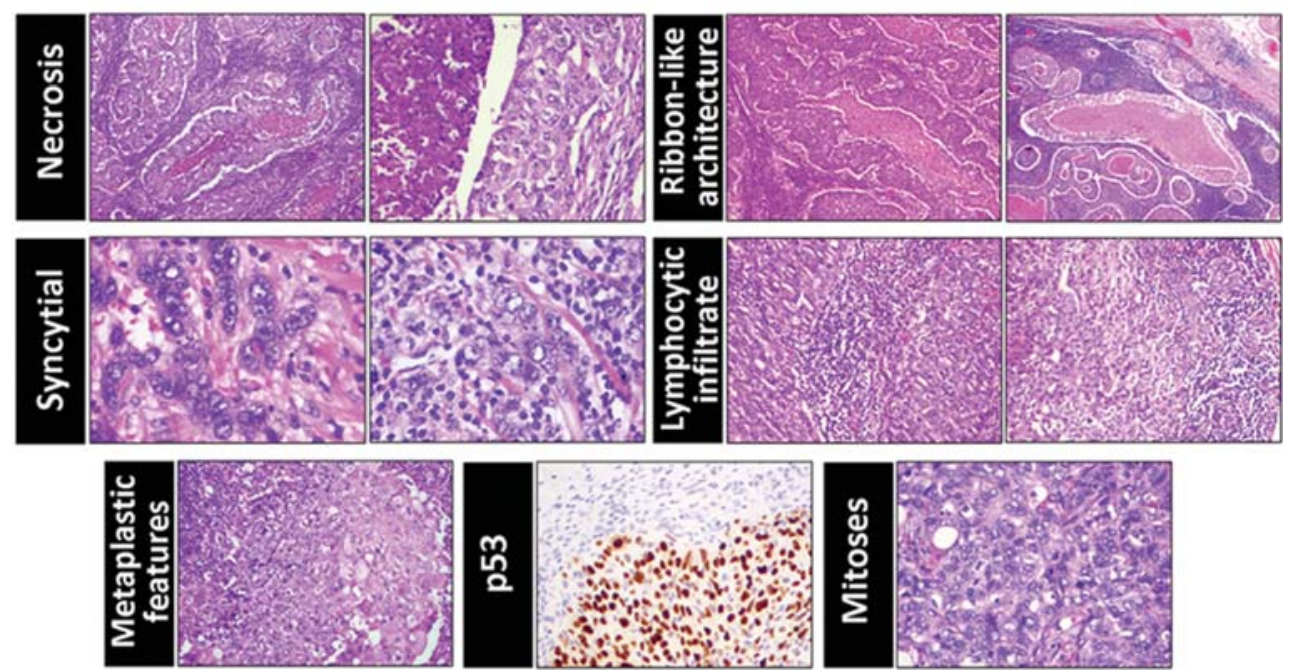

Figure 1. Immunophenotype and morphological features of basal/HER2+ breast carcinomas. (A) Examples of immunohistochemical stainings of HER2 ${ }^{+}$and CK5/CK6 in basal/HER2 ${ }^{+}$breast carcinomas (original magnifications, $x 400$ left and right panels and x200 middle panels). (B) Examples of morphological features of basal/HER2 ${ }^{+}$breast carcinomas. All basal/HER2 ${ }^{+}$tumors showed an overall modified Scarff-Bloom-Richardson grade of 3 (i.e. solid structure and high density of tumor cells). Some basal/HER2+ tumors showed a pushing border and some degree of a stromal lymphocytic infiltrate at the tumor edge while others demonstrated histological features similar to those described for atypical medullary carcinoma (i.e. predominantly pushing border, syncytial arrangement of tumor cells and marked stromal lymphocytic infiltrate). Regions of ribbon-like architecture with associated central necrosis were also observed; despite a high mitotic rate was identified in all basal/HER2 ${ }^{+}$breast carcinomas, apoptotic tumor cells were numerous in cases showing geographic or comedo necrosis. Metaplastic components including squamous metaplasia were found in some cases.

a first attempt to pre-clinically discover novel biomarkers that could be employed for functionally studying HER2+/ basal-like breast carcinomas with primary refractoriness to trastuzumab, we took advantage of the intrinsic trastuzumab resistance in a cell line isolated from the pleural fluid of a HER2-positive breast cancer patient with progressive disease on Tzb (i.e. JIMT-1) (Fig. 2). Despite its paradoxical HER2 gene amplification, ER/PR-negative JIMT-1 cells have repeatedly been classified as basal-like breast cancer cells mainly due to the fact that they express both basal CK5/ CK14 and luminal CK8/CK18 cytokeratins $(11,13)$. Highresolution genomic profiles have confirmed that JIMT-1 cells have the closest resemblance to the HER2+ breast cancer subtype but also to the stem/progenitor (basal-like) breast tumors (23). Indeed, HER2-gene amplified JIMT-1 cells display several of the molecular features distinctively associated with the unique histogenesis of basal-like breast carcinomas including a strong positivity for p53 and high levels of vimentin and p63 expression. Here, we performed low-scale proteomic analyses to obtain pathway-focused molecular signatures in JIMT-1 cells as well as in SKBR3 cells, a widely-used in vitro model of HER2 overexpression exquisitely sensitive to the growth inhibitory actions of trastuzumab.

Growth factor secretome signature. We applied antibodybased array systems to identify growth factors/secreted growth factor receptor signatures in HER2 ${ }^{+}$(basal-like) JIMT-1 breast cancer cells when compared to HER2+ (luminal) SKBR3 breast cancer cells (Fig. 3A). JIMT-1 cells drastically up-regulated, among other ligands belonging to the epidermal growth factor (EGF)-like and fibroblast growth factor (FGF) families, the secretion of amphiregulin and EGF. JIMT-1 cells secreted also enormous amounts of the insulin growth factor binding protein-6 (IGFBP-6) and significantly up-regulated the secretion of the neurotrophins NT-3 and NT-4. Interestingly, we noted a switch between the main platelet-derived growth factor (PDGF) isoform expressed in JIMT-1 (PDGF-AA) and SKBR3 (PDGF-BB) cells, respectively. When compared to SKBR3 cells, pro-angiogenic features were clearly noted in the secretome of JIMT-1 cells as they up-regulated all the isoforms ( $\beta 1, \beta 2$ and $\beta 3)$ of the indirect 


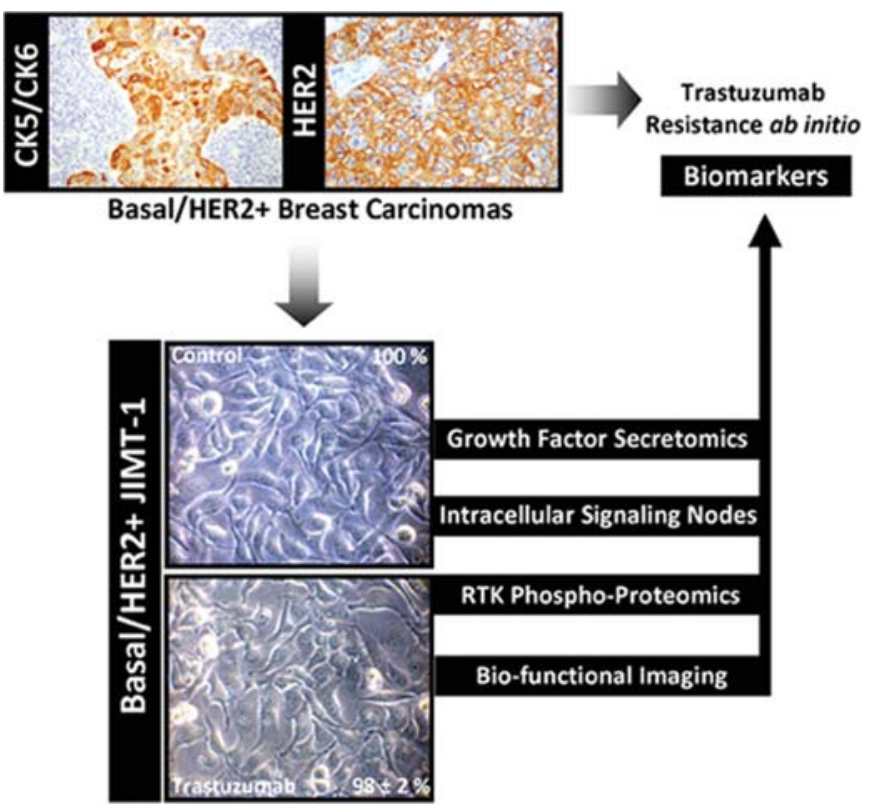

Figure 2. Methodological approach for biomarker discovery in trastuzumabrefractory basal/HER $2^{+}$breast carcinomas. Harris and colleagues demonstrated that HER2+ breast carcinomas expressing genes associated with the basallike phenotype including higher expression of basal cytokeratins were more frequent in the non-responding group of patients receiving pre-operative trastuzumab (14). Here, we performed pathway-focused (low-scale) proteomic analyses as well bio-functional imaging in the HER2 gene-amplified JIMT-1 basal-like breast cancer cell line, which was originally established from a ductal carcinoma pleural metastasis of a 62-year-old breast cancer patient who did not respond to trastuzumab, to delineate basal/HER2+ molecular signatures that may account for refractoriness to HER targeting therapies ab initio.

angiogenic agent TGF (transforming growth factor) as well as the well-recognized angiogenic effector VEGF (vascular endothelial growth factor) and its receptor (VEGF-R2).

Intracellular signaling nodes. We assessed the activation status of convergence points and key regulatory proteins in several signaling pathways controlling cellular events such as growth and differentiation, energy homeostasis and the response to stress and inflammation (Fig. 3B). We took advantage of the CST's PathScan ${ }^{\circledR}$ Signaling Nodes MultiTarget Sandwich ELISA kit (Cell Signaling Technology, Inc.) to simultaneously assess, in a semi-quantitative manner, the endogenous levels of AKT1, phospho-AKT1 (Ser473), phospho-MEK1 (Ser217/221), phospho-p38 MAPK (Thr180/ Tyr182), phospho-Stat3 (Tyr705) and phospho-NF-кB p65 (Ser536) in Tzb-naive SKBR3 cells and Tzb-resistant JIMT-1 cells. JIMT-1 cells not only expressed significantly lower levels of total AKT1 protein but they displayed further a very low phospho-activity profile in all convergence signaling points regulated by MEK1, p38 MAPK and Stat3 proteins when compared to trastuzumab-sensitive SKBR3 cells. Trastuzumab treatment failed to significantly alter constitutive low levels of phospho-AKT1/-MEK1/-p38 MAPK and -Stat3 in JIMT-1 cells (data not shown). Interestingly, JIMT-1 cells displayed a drastic overactivation of NF- $\mathrm{KB}$ p65 when compared to SKBR3 cells. High levels of NF- $\kappa$ B p65 remained unaltered following treatment of JIMT-1 cells with trastuzumab (data not shown).
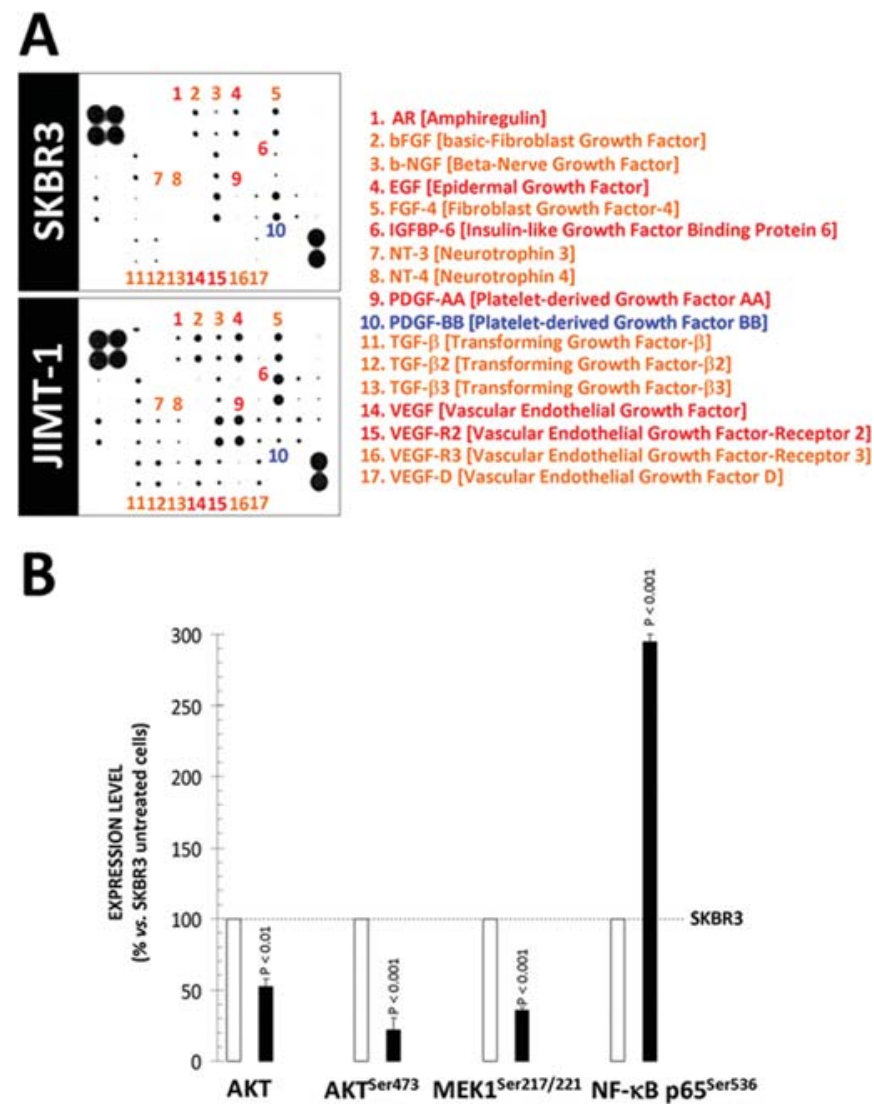

Figure 3. Growth factor secretomics and activation status of intracellular signaling nodes in basal/HER2 ${ }^{+}$JIMT-1 cells. (A) Conditioned media from SKBR3 and JIMT-1 cells were assayed using the RayBio human growth factors/secreted growth factor receptors array 1 (RayBiotech, Inc.) as per manufacturer's instructions. Figure shows two representative arrays $(n=2)$. Blue color indicates significant down-regulation as compared to SKBR3 cells; orange and red colors indicate moderate ( $>5$-fold) and strong $(>10$-fold) up-regulation as compared to SKBR3 cells. (B) Lysates from SKBR3 and JIMT-1 cells were assayed at a protein of $0.5 \mathrm{mg} / \mathrm{ml}$ using the PathScan Signaling Nodes Multi-Target Sandwich ELISA Kit no. 7272 (Cell Signaling Technology, Inc.) as per manufacturer's instructions. The absorbance readings at $450 \mathrm{~nm}$ were normalized to those obtained in untreated control cells cultured strictly in parallel. Results are means (columns) and 95\% confidence intervals (bars) of two independent experiments made in duplicate.

Receptor tyrosine kinase (RTK) phospho-proteome signature. We evaluated whether activation/deactivation of HER network-related receptor tyrosine kinases (RTKs) might significantly differ in trastuzumab-refractory JIMT-1 cells and trastuzumab-naive SKBR3 cells. To simultaneously assess the activation status of multiple RTKs, we took advantage of the human phospho-RTK array, a semi-quantitative tool able to simultaneously identify the phosphorylation status of 42 RTKs in a rapid and sensitive manner (Fig. 4A). In untreated SKBR3 breast cancer cells, the three members of the HER network HER1 (EGFR), HER2 and HER3 were likewise highly phosphorylated, and HER2 phosphorylation was completely suppressed in the presence of trastuzumab. Trastuzumab treatment slightly reduced HER3 trans-activation and slightly increased the phosphorylation status of insulin receptor (IR) and insulin growth factor-1 receptor (IGF-1R). In marked contrast, phospho-RTK profiling of cell lysates from untreated JIMT-1 cells clearly revealed that, unlike HER1 (EGFR) activity, the constitutive status of 


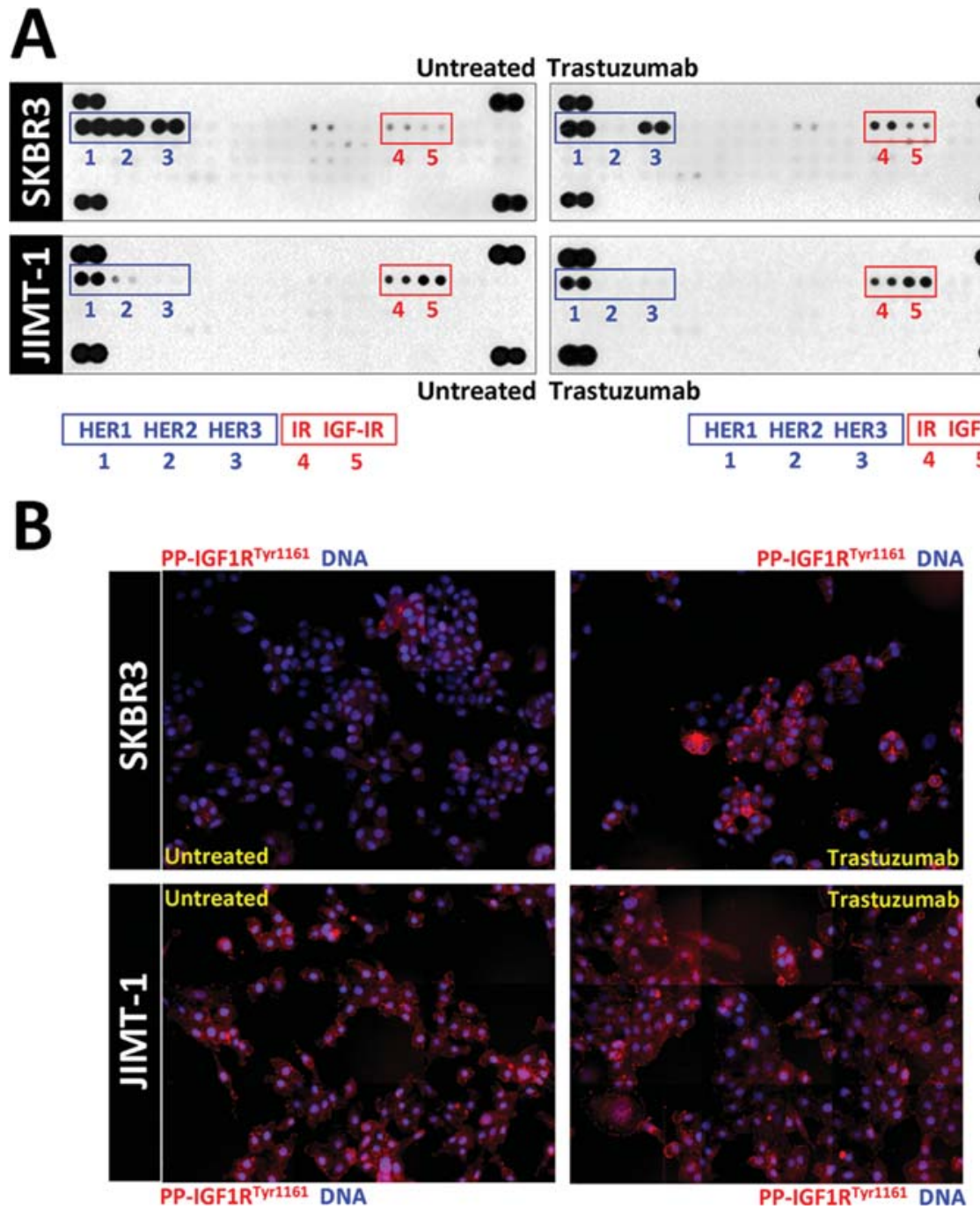

Figure 4. Phospho-proteome profiling of receptor tyrosine kinases in basal/HER2 JIMT-1 cells. (A) Phospho-proteome profiling of SKBR3 and JIMT-1 cells prior and after treatment with trastuzumab $(100 \mu \mathrm{g} / \mathrm{ml})$ for $48 \mathrm{~h}$. Total cell lysates $(500 \mu \mathrm{g})$ were incubated with membranes of the human phospho-RTK (42 different RTKs) as per manufacturer's instructions (Proteome Profiler; R\&D Systems). Phospho-RTK Array data were developed on X-ray film following exposure to chemiluminescent reagents. Figure shows representative phospho-proteome analyses. Equivalent results were obtained in three independent experiments. (B) Images show representative whole populations of SKBR3 and JIMT-1 cells growing in the absence or presence of trastuzumab (100 $\mu \mathrm{g} / \mathrm{ml})$ that were captured in individual wells using different channels for PP-IGF-1R ${ }^{\text {Tyr1161 }}$ (red) and Hoechst 33258 (blue) as a $4 x 4$ montage with a $x 20$ objective on BD Pathway 855 Bioimager System, and merged using BD Attovision software.

HER2 and HER3 phosphorylation was markedly decreased compared to that observed in untreated SKBR3 cells. While treatment trastuzumab was sufficient to abrogate the slight HER2 phosphorylation, JIMT-1 cells exhibited a trastuzumabrefractory hyperactivation of IR and IGF-1R, thus suggesting that the insulin signaling-related cytoprotective stress response observed in trastuzumab-naive SKBR3 cells is constitutively up-regulated in JIMT-1 cells.

Bio-functional imaging of phospho-IGF-1R in basal/HER ${ }^{+}$ JIMT-1 cells. Because the hyper-phosphorylated status of IR/IGF-1R, which could compensate for attenuated HER2 signaling in trastuzumab-refractory JIMT-1 cells, remained unaltered in the presence of trastuzumab, and because costaining experiments failed to demonstrate co-localization of PP-IGF-1R Tyr1161 and HER2 (data not shown), we speculated that signaling emanating from the insulin/IGF-1 transduction cascade could take place in separated sub-cellular compart- ments functionally unrelated to HER signaling platforms in trastuzumab-refractory JIMT-1 cells. In agreement with this notion, we observed striking accumulations of PP-IGR$\mathrm{IR}^{\mathrm{Tyr} 1161}$ at numerous and distinct cell membrane-associated plaques when performing high-content immunofluorescence microscopy studies to visualize sub-cellular accumulation of the active form of IGF-IR before and after treatment with trastuzumab (Fig. 4B). This phenomenon, however, occurred exclusively in trastuzumab-resistant JIMT-1 cells but not in trastuzumab-sensitive SKBR3 cells.

Co-staining experiments with antibodies directed against phosphorylated IGF-1R and the cytoskeletal protein F-actin strongly suggested that trastuzumab-refractory JIMT-1 cells appear to exhibit a significant reorganization of the cytoskeleton when compared that observed in trastuzumab-sensitive SKBR3 cells. Whereas trastuzumab-enhanced IGF-1R phosphorylation formed continuous lines along the borders of cell-cell contacts in SKBR3 cells (where F-actin was also 


\section{A}
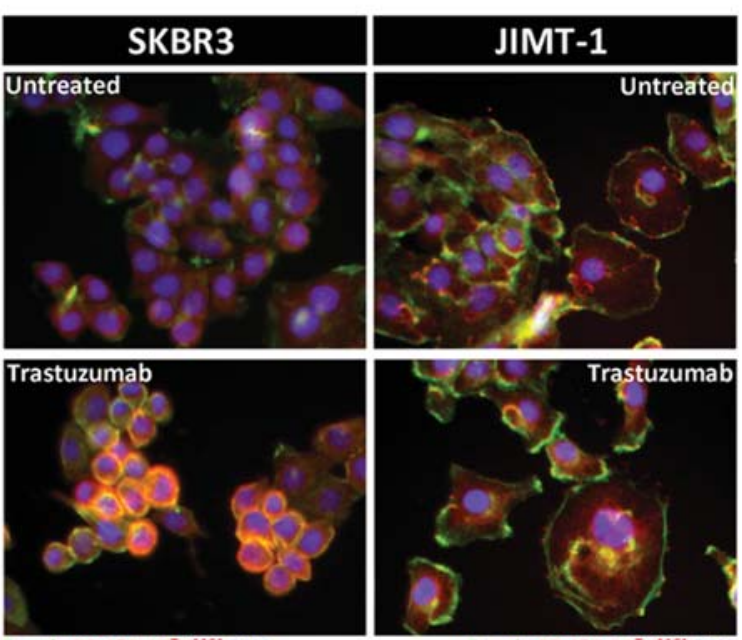

B

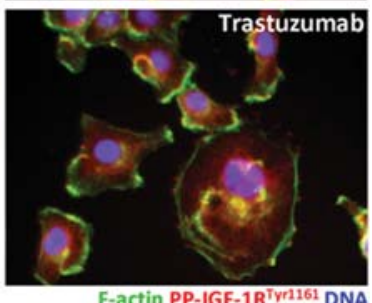

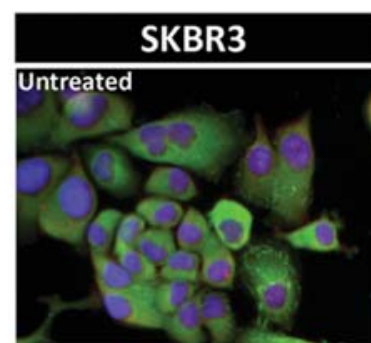

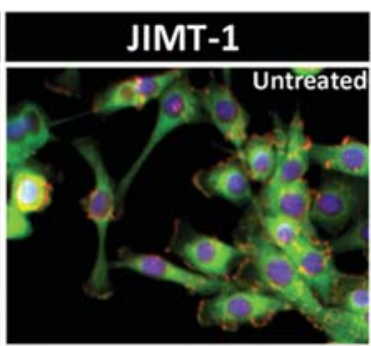

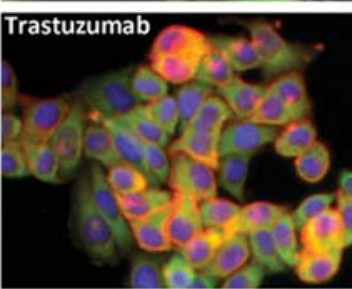

Vinculin PP-IGF-1R ${ }^{\mathrm{Ty}+1161}$ DNA

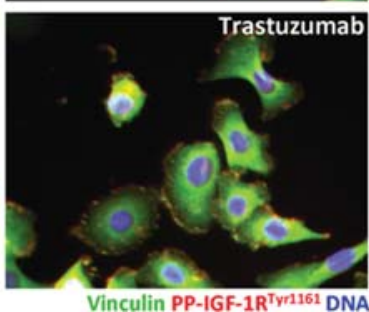

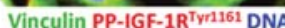

Figure 5. Cytoskeleton organization and focal adhesion distribution in basal/HER2+ JIMT-1 cells. After fixation and permeabilization, cellular codistribution of PP-IGF1R ${ }^{\text {Tyr } 1161}$ and F-actin (A) or vinculin (B) was assessed following staining with anti-PP-IGF1R ${ }^{\mathrm{Tyr} 1161}$, anti-F-actin and anti-vinculin antibodies, as specified, and Hoechst 33258 for nuclear counterstaining. Images show representative portions of SKBR3 and JIMT-1 cells cultured in the absence or presence of trastuzumab $(100 \mu \mathrm{g} / \mathrm{ml})$ and captured in different channels for PP-IGF-1R $\mathrm{R}^{\mathrm{T} y \mathrm{r} 161}$ (red), F-actin (green), vinculin (green) and Hoechst 33258 (blue) with a x20 objective, and merged on BD Pathway 855 Bioimager System using BD Attovision software.

localized extensively), JIMT-1 cells had punctuated instead of continuous distribution of PP-IGF-1R $\mathrm{R}^{\mathrm{Ty} 1161}$ at cell-cell borders with $\mathrm{F}$-actin concentrating within membrane protrusions (Fig. 5A). Indeed, co-staining experiments with the cytoskeletal protein vinculin confirmed further that PP-IGF1RTyr1161 somewhat concentrated at the edges of profuse and numerous focal adhesions-like structures in the cell membrane of JIMT-1 cells (Figs. 5B and 6A). These phenomena might relate to changes in the cell migratory behavior of JIMT-1 cells. In vitro scratch wound healing assays showed a significantly faster migration rate in JIMT-1 basal-like cells compared to the very low locomotory activity of SKBR3 luminal cells (data not shown). Perhaps more importantly, the highly migratogenic phenotype of JIMT-1 cells took place irrespective of the absence or presence of trastuzumab (Fig. 6B).
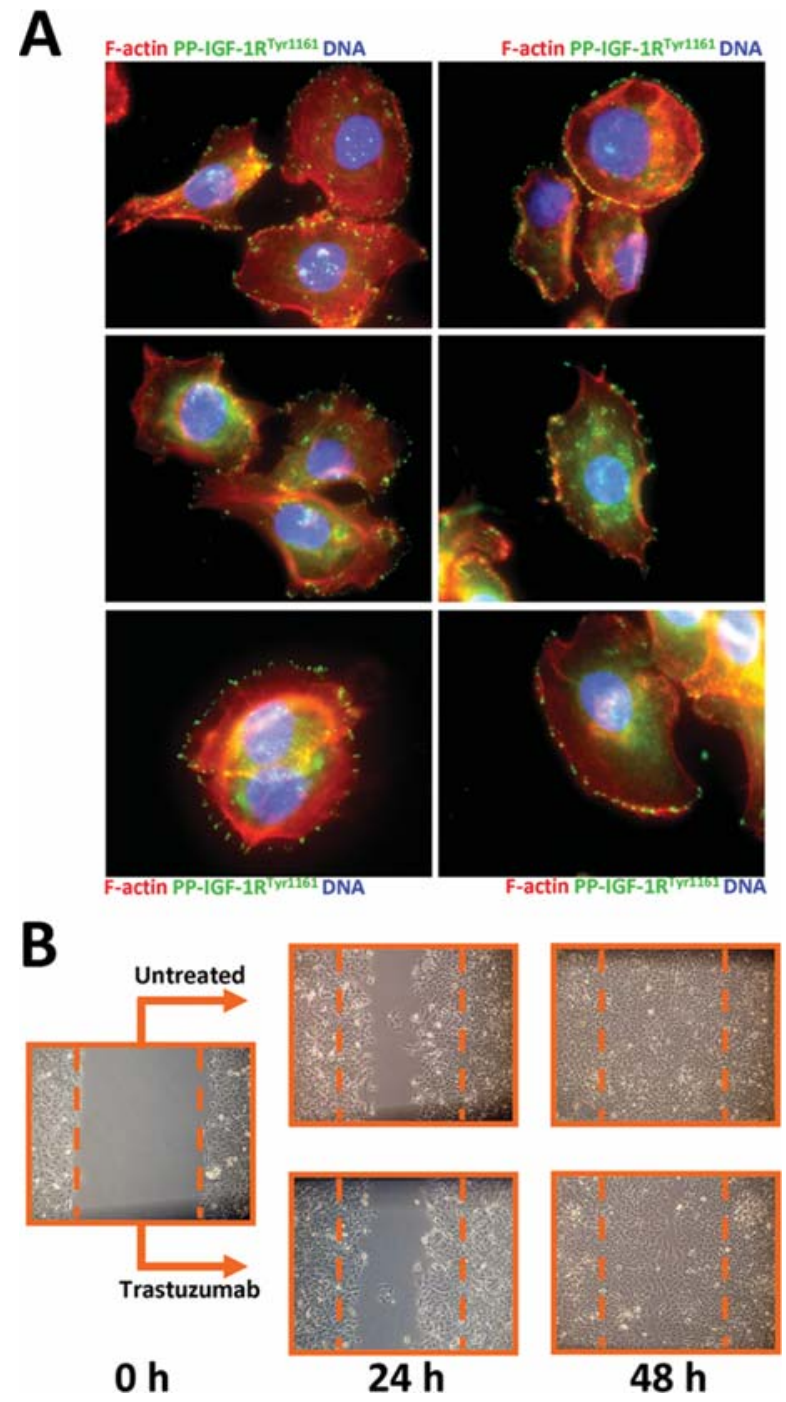

Figure 6. A correlation between activated IGF-1R at focal adhesions and trastuzumab-refractory migratogenic phenotype in basal/HER2 ${ }^{+}$JIMT-1 cells. (A) After fixation and permeabilization, cellular co-distribution of PPIGF1R ${ }^{\text {Tyr1161 }}$ and F-actin was assessed following staining with anti-PP$\mathrm{IGF}^{\mathrm{T}} \mathrm{R}^{\mathrm{Ty} 1161}$ and anti-F-actin antibodies, as specified, and Hoechst 33258 for nuclear counterstaining. Images show representative portions of JIMT-1 cells captured in different channels for PP-IGF-1R $\mathrm{R}^{\mathrm{Tyr} 1161}$ (green), F-actin (red) and Hoechst 33258 (blue) with a x40 objective, and merged on BD Pathway 855 Bioimager System using BD Attovision software. (B) Figure shows representative micrographs of JIMT-1 cells wound healing assays 0, 24 and $48 \mathrm{~h}$ after incisions of confluent JIMT-1 cells cultured in the absence or presence of trastuzumab $(100 \mu \mathrm{g} / \mathrm{ml})$.

\section{Discussion}

It is well recognized that breast cancer disease exhibits a heterogeneous clinical, histological and molecular diversity. Gene expression profiling and biomarker studies have repeatedly shown, at least, five distinct molecular subtypes of breast cancer: luminal $\mathrm{A}\left(\mathrm{ER}^{+}\right.$and/or $\left.\mathrm{PR}^{+}, \mathrm{HER} 2^{-}\right)$, luminal $\mathrm{B}$ $\left(\mathrm{ER}^{+}\right.$and/or $\left.\mathrm{PR}^{+}, \mathrm{HER} 2^{+}\right)$, HER2-overexpressing (ER-/PR', $\mathrm{HER}^{+}{ }^{+}$), basal-like (ER-/PR- HER $2^{-}, \mathrm{CK} 5 / \mathrm{CK}^{-}{ }^{+}$and/or $\mathrm{EGFR}^{+}$) and normal breast-like tumors. Since different keratins predominate in the different lineages of the normal breast, with CK18 and CK19 being expressed in the luminal cells and CK5/CK6 and CK14 in basal/myoepithelial cells, breast carcinomas can also be broadly categorized into two 
classes: those that express luminal keratins (luminal-type) and those that express stratified epithelial keratins (basallike). In this scenario, it has been suggested that a subset of HER2+ breast carcinomas should originate from luminal cells with HER2 gene amplification and HER2 protein overexpression being a relatively late event in $\mathrm{HER} 2^{+}$breast cancer tumorigenesis. However, it is well recognized that HER2 overexpression frequently occurs in premalignant lesions, such as DCIS, and overexpression of HER2 significantly increases the fraction of breast cancer cells positive for ALDH1 (aldehyde dehydrogenase), a marker of normal and cancerous human mammary epithelial cells with stem/ progenitor properties (27). Our current confirmation of the presence of basal cytokeratins (i.e. CK5/CK6) in HER2+ breast carcinomas, thus denoting a basal/HER2 ${ }^{+}$phenotype that has been shown recently to exhibit poorer survival than basal-like phenotype in hormone receptor-negative invasive breast carcinomas (24), therefore raises the possibility that these tumors may originate from stem cells that subsequently undergo variable degrees of basal and luminal differentiation. Given that prognostic features of the basal/HER $2^{+}$phenotype might be intrinsically different to those found in either HER2+ or basal-like breast carcinomas, it would be reasonable to suggest that this group of tumors might require different treatment strategies. Indeed, the presence of a basal/HER2+ phenotype may largely explain the pioneer finding that a particular HER2+ tumor phenotype that was more likely to express genes associated with the basal-like phenotype, including higher expression of basal keratins (CK5, CK14, CK15 and CK17), was more frequent in the non-responding group of patients receiving pre-operative trastuzumab (14). Because a better understanding of the genes and pathways of basal/HER2 ${ }^{+}$breast cancer cells may allow development of additional approaches to the successful treatment of HER2+ breast cancer, we have explored the occurrence of novel proteomic signatures in JIMT-1 breast cancer cells, a unique model to explore de novo mechanisms of resistance to trastuzumab in HER 2 gene-amplified breast cancer cells with a basal-like phenotype.

Harris and colleagues reported that HER2 ${ }^{+}$tumors with de novo refractoriness to trastuzumab expressed higher levels of growth factors including IGF-1, hepatocyte growth factor, PDGF and pleiotrophin (14). Although virtually nothing is known about the paracrine/autocrine/intracrine actions of growth factors in the efficacy of HER targeting agents, we are beginning to accumulate evidence that the efficacy of anticancer drugs that block one or several members of the HER network may be compromised by the presence of EGFrelated ligands (28-30). Motoyama and colleagues conducted pioneering work in demonstrating that the growth-inhibitory effects of HER1/HER2 tyrosine kinase inhibitors (TKIs) are significantly attenuated in the presence of exogenous heregulin, which is a high-affinity combinatorial ligand for HER3 and HER4 receptors in breast cancer cells (31). Within the same context of HER-targeting inhibitors, it has been observed that breast cancer cells with acquired resistance to trastuzumab express higher endogenous levels of EGF-related ligands as compared with parental, trastuzumab-sensitive cells (32). We and others have demonstrated that the growthinhibitory effects of HER inhibitors indeed correlate with expression levels of certain HER ligands $(29,30)$. JIMT-1 breast cancer cells not only exhibit primary resistance to trastuzumab but demonstrate further cross-resistance to several HER2-inhibiting drugs including the HERdimerization inhibitor antibody pertuzumab (2C4) and the small molecule HER TKIs CI1033 (canertinib), ZD1839 (gefitinib) and lapatinib $(11,33)$. It is noteworthy that, when compared to HER2+ SKBR3 cells, a luminal breast cancer model exquisitely sensitive to HER targeting agents including trastuzumab, gefitinib and lapatinib, the JIMT-1 secretome accumulated significantly higher amounts of several growth factors such as amphiregulin and EGF (two high-affinity ligands of EGFR (HER1), a growth factor receptor commonly found overexpressed in basal-like breast tumors) as well as several isoforms of the pro-angiogenic factors TGF 3 and VEGF. Most of these secreted factors are important components of the tumor-stromal microenvironment and, in vivo, they may play yet to be explored mitogenic and chemotactics that may account for resistance to trastuzumabbased therapy.

Extensive preclinical and limited clinical data have suggested that IGF-1R is associated with acquired (i.e. secondary) auto-resistance to trastuzumab (1-4,6,7). In a scenario of trastuzumab resistance $a b$ initio, Harris and colleagues reported that finding either IGF- 1 gene overexpression by array or IGF-1R protein expression on the membrane in trastuzumab-resistant tumors might suggest the presence of an autocrine loop in which IGF-1R signaling actively mediates de novo resistance to trastuzumab therapy (14). Here, by using low-scale phospho-proteomic analyses, we reveal that trastuzumab-refractory basal/HER2+ JIMT-1 cells display a reduced signaling through HER2 that is accompanied by constitutive and trastuzumab-insensitive hyperactivation of the alternative signaling cascade IR/IGF$1 \mathrm{R}$. Initially, the significance of this finding was unclear because constitutive hyperactivation of IR/IGF-1R failed to associate with an enhanced activation of both mitogenactivated protein kinase (MAPK) and AKT pathways, which are critical for HER2 signal transduction. Rather, IR/IGF-1R hyperactivation in basal/HER2 ${ }^{+}$breast carcinomas may release these tumors from dependence on HER2 for proliferation and survival through two novel pathways. On the one hand, JIMT-1 cells drastically oveactivated RelA, the prosurvival subunit of NF- $\kappa \mathrm{B}$, a recently discovered cytoprotective stress response that confers resistance to the dual HER1/HER2 TKI lapatinib (34). On the other hand, unlike uniform localization of activated IFG-1R in the cell membrane of trastuzumabtreated luminal/HER2+ SKBR3 cells, activated IGF-1R mainly localized at numerous and profuse focal adhesions, like structures in basal/HER2+ JIMT-1 cells, which exhibited a significant cytoskeleton reorganization accompanied by an exacerbated trastuzumab-refractory migratogenic phenotype. Our findings support the notion that basal-like breast carcinomas exhibit high motility and invasive properties as well as the accumulating evidence that the IGF/IGFR transduction node functions not only as mitogenic factor but also as promoter of cell motility (35-38). It has been shown that activated IGF-1R associates to and activates integrins at the focal adhesion contacts, probably acting through cytoskeletal proteins such as vinculin. As a consequence of cell adhesion, 
autophosphorylation of focal adhesion kinase (FAK) takes place and focal adhesions are assembled. Our current biofunctional imaging analyses suggest that this straightforward sequence of events could explain how constitutive IGF-1R hyperactivation may stimulate JIMT-1 cell migration. Recent studies have revealed that trastuzumab treatment is sufficient to abrogate lamellipodia formation and MMP-independent cell migration in HER2 gene-amplified breast cancer cells (39). Moreover, intracellular signaling emanating from FAK may mediate cell survival via $\mathrm{NF}-\mathrm{\kappa B}$ (40) while removal or inhibition of the laminin-integrin-CD151-FAK axis markedly sensitizes HER2+ breast cancer cells to anti-HER2 agents including trastuzumab and lapatinib (41). Forthcoming studies should elucidate if the biochemical mechanisms by which constitutively active IGF-1R allows a highly-migratogenic phenotype in basal/HER $2^{+}$breast cancer cells can relate also to trastuzumab refractoriness via downstream activation of pro-survival/anti-apoptotic signaling such as NF-кB.

In summary, our current definition of novel secretomic and phospho-proteomic signatures in a basal/HER2 ${ }^{+}$breast carcinoma model may provide new insights into the ultimate molecular mechanisms underlying de novo refractoriness to HER-targeted therapies including trastuzumab. Although both the secretomic and phospho-proteomic features of JIMT-1 cells might eventually provide new targets for circumventing de novo resistance to trastuzumab in HER 2+ breast carcinomas, their ultimate biological function regarding trastuzumab efficacy should be validated by employing pharmacological and/or siRNA-based approaches. Immunohistochemical and/or microarray-based studies in breast cancer tissues should validate the notion that identification of progenitor (basal-like) phenotypes within HER2+ breast carcinomas may provide a rapid means to define subgroups of HER2-over-expressing breast cancer patients likely to display resistance to HER targeting therapies ab initio.

\section{Acknowledgments}

Alejandro Vazquez-Martin is the recipient of a Sara Borrell post-doctoral contract (CD08/00283, Ministerio de Sanidad y Consumo, Fondo de Investigación Sanitaria (FIS), Spain). Work at the Menendez Laboratory is supported by the Instituto de Salud Carlos III (Ministerio de Sanidad y Consumo, Fondo de Investigación Sanitaria (FIS), Spain, Grants CP05-00090 and PI06-0778 and RD06-0020-0028), the Fundación Científica de la Asociación Española Contra el Cáncer (AECC, Spain), and by the Ministerio de Ciencia e Innovación (SAF2009-11579, Plan Nacional de I+D+ I, MICINN, Spain). This work was also supported by a charity collection organized by the Fundació Roses Contra el Cáncer (Roses, Girona, Catalonia).

\section{References}

1. Nahta R and Esteva FJ: HER-2-targeted therapy: lessons learned and future directions. Clin Cancer Res 9: 5078-5084, 2003.

2. Nahta R and Esteva FJ: Herceptin: mechanisms of action and resistance. Cancer Lett 232: 123-138, 2006.

3. Nahta R, Yu D, Hung MC, Hortobagyi GN and Esteva FJ: Mechanisms of disease: understanding resistance to HER2targeted therapy in human breast cancer. Nat Clin Pract Oncol 3: 269-280, 2006.
4. Nahta R and Esteva FJ: Trastuzumab: triumphs and tribulations. Oncogene 26: 3637-3643, 2007.

5. Vogel CL, Cobleigh MA, Tripathy D, Gutheil JC, Harris LN, Fehrenbacher L, Slamon DJ, Murphy M, Novotny WF, Burchmore M, Shak S, Stewart SJ and Press M: Efficacy and safety of trastuzumab as a single agent in first-line treatment of HER2-overexpressing metastatic breast cancer. J Clin Oncol 20: 719-726, 2002 .

6. Valabrega G, Montemurro F and Aglietta M: Trastuzumab: mechanism of action, resistance and future perspectives in HER2-overexpressing breast cancer. Ann Oncol 18: 977-984, 2007.

7. Nahta R and Esteva FJ: HER2 therapy: molecular mechanisms of trastuzumab resistance. Breast Cancer Res 8: 215, 2006.

8. Chen FL, Xia W and Spector NL: Acquired resistance to small molecule ErbB2 tyrosine kinase inhibitors. Clin Cancer Res 14: 6730-6734, 2008.

9. Spector NL and Blackwell KL: Understanding the mechanisms behind trastuzumab therapy for human epidermal growth factor receptor 2-positive breast cancer. J Clin Oncol 27: 5838-5847, 2009.

10. Esteva FJ, Yu D, Hung MC and Hortobagyi GN: Molecular predictors of response to trastuzumab and lapatinib in breast cancer. Nat Rev Clin Oncol 7: 98-107, 2010.

11. Tanner M, Kapanen AI, Junttila T, Raheem O, Grenman S, Elo J, Elenius $\mathrm{K}$ and Isola $\mathrm{J}$ : Characterization of a novel cell line established from a patient with Herceptin-resistant breast cancer. Mol Cancer Ther 3: 1585-1592, 2004.

12. Nagy P, Friedländer E, Tanner M, Kapanen AI, Carraway KL, Isola $J$ and Jovin TM: Decreased accessibility and lack of activation of ErbB2 in JIMT-1, a herceptin-resistant, MUC4expressing breast cancer cell line. Cancer Res 65: 473-482, 2005.

13. Pályi-Krekk Z, Barok M, Isola J, Tammi M, Szöllosi J and Nagy P: Hyaluronan-induced masking of ErbB2 and CD44enhanced trastuzumab internalisation in trastuzumab resistant breast cancer. Eur J Cancer 43: 2423-2433, 2007.

14. Harris LN, You F, Schnitt SJ, Witkiewicz A, Lu X, Sgroi D, Ryan PD, Come SE, Burstein HJ, Lesnikoski BA, Kamma M, Friedman PN, Gelman R, Iglehart JD and Winer EP: Predictors of resistance to preoperative trastuzumab and vinorelbine for HER2positive early breast cancer. Clin Cancer Res 13: 1198-1207, 2007.

15. Lesniak D, Xu Y, Deschenes J, Lai R, Thoms J, Murray D, Gosh S, Mackey JR, Sabri S and Abdulkarim B: Beta1-integrin circumvents the antiproliferative effects of trastuzumab in human epidermal growth factor receptor-2-positive breast cancer. Cancer Res 69: 8620-8628, 2009

16. Perou CM, Sørlie T, Eisen MB, van de Rijn M, Jeffrey SS, Rees CA, Pollack JR, Ross DT, Johnsen H, Akslen LA, Fluge O, Pergamenschikov A, Williams C, Zhu SX, Lønning PE, Børresen-Dale AL, Brown PO and Botstein D: Molecular portraits of human breast tumours. Nature 406: 747-752, 2000 .

17. Sorlie T, Perou CM, Tibshirani R, Aas T, Geisler S, Johnsen H, Hastie T, Eisen MB, van de Rijn M, Jeffrey SS, Thorsen T, Quist H, Matese JC, Brown PO, Botstein D, Eystein Lønning P and Børresen-Dale AL: Gene expression patterns of breast carcinomas distinguish tumor subclasses with clinical implications. Proc Natl Acad Sci USA 98: 10869-10874, 2001.

18. Sorlie T, Tibshirani R, Parker J, Hastie T, Marron JS, Nobel A, Deng S, Johnsen H, Pesich R, Geisler S, Demeter J, Perou CM, Lønning PE, Brown PO, Børresen-Dale AL and Botstein D: Repeated observation of breast tumor subtypes in independent gene expression data sets. Proc Natl Acad Sci USA 100: 8418-8423, 2003.

19. Rakha EA, Reis-Filho JS and Ellis IO: Basal-like breast cancer: a critical review. J Clin Oncol 26 :2568-2581, 2008.

20. Fadare $\mathrm{O}$ and Tavassoli FA: Clinical and pathologic aspects of basal-like breast cancers. Nat Clin Pract Oncol 5: 149-159, 2008.

21. Laakso M, Tanner M, Nilsson J, Wiklund T, Erikstein B, Kellokumpu-Lehtinen P, Malmström P, Wilking N, Bergh J and Isola J: Basoluminal carcinoma: a new biologically and prognostically distinct entity between basal and luminal breast cancer. Clin Cancer Res 12: 4185-4191, 2006.

22. Livasy CA, Karaca G, Nanda R, Tretiakova MS, Olopade OI, Moore DT and Perou CM: Phenotypic evaluation of the basallike subtype of invasive breast carcinoma. Mod Pathol 19: 264-271, 2006. 
23. Jönsson G, Staaf J, Olsson E, Heidenblad M, VallonChristersson J, Osoegawa K, de Jong P, Oredsson S, Ringnér M, Höglund $\mathrm{M}$ and Borg A: High-resolution genomic profiles of breast cancer cell lines assessed by tiling BAC array comparative genomic hybridization. Genes Chromosomes Cancer 46: 543-558, 2007

24. Liu H, Fan Q, Zhang Z, Li X, Yu H and Meng F: Basal-HER2 phenotype shows poorer survival than basal-like phenotype in hormone receptor-negative invasive breast cancers. Hum Pathol 39: 167-174, 2008

25. Yehiely F, Moyano JV, Evans JR, Nielsen TO and Cryns VL: Deconstructing the molecular portrait of basal-like breast cancer. Trends Mol Med 12: 537-544, 2006.

26. Sarrió D, Rodriguez-Pinilla SM, Hardisson D, Cano A, MorenoBueno G and Palacios J: Epithelial-mesenchymal transition in breast cancer relates to the basal-like phenotype. Cancer Res 68: 989-997, 2008.

27. Bedard PL, Cardoso F and Piccart-Gebhart MJ: Stemming resistance to HER-2 targeted therapy. J Mammary Gland Biol Neoplasia 14: 55-66, 2009.

28. Smith BL, Chin D, Maltzman W, Crosby K, Hortobagyi GN and Bacus SS: The efficacy of Herceptin therapies is influenced by the expression of other erbB receptors, their ligands and the activation of downstream signalling proteins. Br J Cancer 91: 1190-1194, 2004.

29. Menendez JA and Lupu R: Transphosphorylation of kinasedead HER 3 and breast cancer progression: a new standpoint or an old concept revisited? Breast Cancer Res 9: 111, 2007.

30. Ferrer-Soler L, Vazquez-Martin A, Brunet J, Menendez JA, De Llorens R and Colomer R: An update of the mechanisms of resistance to EGFR-tyrosine kinase inhibitors in breast cancer: Gefitinib (Iressa) induced changes in the expression and nucleocytoplasmic trafficking of HER-ligands (Review). Int J Mol Med 20: 3-10, 2007.

31. Motoyama AB, Hynes NE and Lane HA: The efficacy of ErbB receptor-targeted anticancer therapeutics is influenced by the availability of epidermal growth factor-related peptides. Cancer Res 62: 3151-3158, 2002.

32. Ritter CA, Perez-Torres M, Rinehart C, Guix M, Dugger T, Engelman JA and Arteaga CL: Human breast cancer cells selected for resistance to trastuzumab in vivo overexpress epidermal growth factor receptor and ErbB ligands and remain dependent on the ErbB receptor network. Clin Cancer Res 13: 4909-4919, 2007.
33. Köninki K, Barok M, Tanner M, Staff S, Pitkänen J, Hemmilä P, Ilvesaro $\mathrm{J}$ and Isola $\mathrm{J}$ : Multiple molecular mechanisms underlying trastuzumab and lapatinib resistance in JIMT-1 breast cancer cells. Cancer Lett 294: 211-219, 2010.

34. Xia W, Bacus S, Husain I, Liu L, Zhao S, Liu Z, Moseley MA 3rd, Thompson JW, Chen FL, Koch KM and Spector NL: Resistance to ErbB2 tyrosine kinase inhibitors in breast cancer is mediated by calcium-dependent activation of RelA. Mol Cancer Ther 9: 292-299, 2010.

35. Mañes S, Mira E, Gómez-Mouton C, Zhao ZJ, Lacalle RA and Martínez-A C: Concerted activity of tyrosine phosphatase SHP-2 and focal adhesion kinase in regulation of cell motility. Mol Cell Biol 19: 3125-3135, 1999.

36. Zhang X, Lin M, van Golen KL, Yoshioka K, Itoh K and Yee D: Multiple signaling pathways are activated during insulin-like growth factor-I (IGF-I) stimulated breast cancer cell migration. Breast Cancer Res Treat 93: 159-168, 2005.

37. Guvakova MA: Insulin-like growth factors control cell migration in health and disease. Int J Biochem Cell Biol 39: 890-909, 2007.

38. Liu W, Bloom DA, Cance WG, Kurenova EV, Golubovskaya VM and Hochwald SN: FAK and IGF-IR interact to provide survival signals in human pancreatic adenocarcinoma cells. Carcinogenesis 29: 1096-1107, 2008

39. Yokotsuka M, Iwaya K, Saito T, Pandiella A, Tsuboi R, Kohno N, Matsubara $\mathrm{O}$ and Mukai K: Overexpression of HER2 signaling to WAVE2-Arp2/3 complex activates MMP-independent migration in breast cancer. Breast Cancer Res Treat, Apr 24, 2010 (E-pub ahead of print)

40. Huang D, Khoe M, Befekadu M, Chung S, Takata Y, Ilic D and Bryer-Ash M: Focal adhesion kinase mediates cell survival via NF-kappaB and ERK signaling pathways. Am J Physiol Cell Physiol 292: C1339-C1352, 2007.

41. Yang XH, Flores LM, Li Q, Zhou P, Xu F, Krop IE and Hemler ME: Disruption of laminin-integrin-CD151-focal adhesion kinase axis sensitizes breast cancer cells to ErbB2 antagonists. Cancer Res 70: 2256-2263, 2010. 